\title{
ÜBERSICHTEN.
}

\section{PSYCHOLOGIE UND PSYCHIATRIE ${ }^{1}$ ). \\ Von \\ Prof. Oswald Bumke. \\ Direktor der psychiatrischen und Nervenklinik in Leipzig.}

Um die psychologischen Grundlagen der Psychiatrie ist ein Streit entstanden, der die gegenwärtige Lage klären und für die Zukunft wichtige Fortschritte anbahnen könnte.

Er ist im wesentlichen dadurch notwendig geworden, daß die experimentelle Psychologie, von der einst gerade die Psychiatrie so besonders viel erhofft hatte, - ich will einmal sagen: die Grenzen ihrer klinischen Brauchbarkeit erreicht hat. Die historische Aufgabe dieser Richtung hat bekanntlich darin bestanden, eine rein spekulative Psychologie, die nicht bloß über das Wesen des Seelischen, sondern auch über seine Erscheinungen ins Blaue hinein philosophierte, abzulösen und zn verdrängen. Diese Aufgabe hat die experimentelle Psychologie glänzend gelöst, aber auch was sie als $Z_{\text {weig der }}$ Physiologie, darüber hinaus geleistet hat, soll hier nicht verkleinert werden. Nur das erscheint uns heute fast unbegreiflich, wie eine ganze Generation im Ernst daran denken konnte, das Psychische mit einer Methode zu erforschen, die allem Seelischen bewußt aus dem Wege ging. Es versteht sich ja doch wohl von selbst, daß physiologische Untersuchungsarten - ganz ähnlich, wie die Versuche, das Psychische im Gehirn zu lokalisieren - uns stets nur bis an die Schwelle der Bewußtseinserscheinungen führen können. Nur körperliche Vorgänge lassen sich messen, zählen und wägen, und nur die von außen kommenden Reize oder die nach außen gehenden körperlichen Reaktionen, die manche seelische Erlebnisse einleiten und abschließen, kann man mit physiologischen Mitteln studieren. Über das, was inzwischen bewußt, was innerlich erlebt wird, vermag immer nur der Auskunft zu geben, bei dem sich diese seelischen Vorgänge abspielen. Kein Streben nach Exaktheit beseitigt den inneren Widerspruch einer Psychologie, die alles Subjektive ausschalten will, - es sei denn, daß man sich von vornherein auf den mechanistischen Standpunkt stellt: "Das Ich ist unrettbar." Damit macht man dann freilich gleich reinen Tisch: man hebt nicht eine, sondern jede Psychologie auf.

Mit der experimentellen Richtung ist aber auch die Associationspsychologie oder, allgemeiner gesprochen, die "Psychologie der Elemente", wie EdUard Spranger sie nennt, zusammengebrochen. Ich weiß, daß ihre anatomischen $B e-$ ziehungen (die zwar nur selten ausdrücklich betont, dafür aber um so häufiger als selbstverständlich vorausgesetzt werden), gerade diese psychologische Richtung im Bewußtsein der Ärzte auch heute noch ziemlich fest verankert halten. Es sieht ja so einfach aus, alle Rätsel des Seelenlebens mit dem verwickelten Bau des Gehirns, das Neben- und Nacheinander psychischer Vorgänge und das Spiel der Motive mit dem Leitungsgesetz und die Geheimnisse des Gedächtnisses mit der Tatsache zu erklären, daß periphere Nerven durch wiederholtes Elektrisieren für den elektrischen Strom ansprechbarer würden. Auch die Erfolge der. Aphasie- und Apraxielehre schienen zunächst ähnliche Grundanschauungen zu stützen. Dazu kam, daß dem anatomisch eingestellten Mediziner die Zerlegung auch des Seelischen in seine „Elemente" als eine beinahe selbstverständliche Vorarbeit für das Studium komplexer psychischer Vorgänge erschien.

Bei kritischer Besinnung ergibt sich aber unzweideutig, daß mit alledem psychologische Erkenntnisse im eigentlichen Sinne niemals gewonnen werden können und tatsächlich auch

1)' Vortrag, gehalten auf der 24. Versammlung mitteldeutscher Psychiater und Neurologen, Dresden, Oktober ż2I. niemals gewonnen worden sind. Die Gehirnanatomie und die Aufdeckung physiclogischer Mechanismen führen uns höchstens wieder in den Vorhof der Psychologie; und daB man durch die Erforschung der sogenannten seelischen Elemente den Grund für die Errichtung höherer Stockwerke des Seelischen legen, ja daB man überhaupt Empfindungen, Wahrnehmungen, Gedanken, Gefühle und Willensimpulse aus dem Strom des psychischen Erlebens fein säuberlich herauskrystallisieren könnte, um nachher durch die Synthese dieser Einzelelemente das Bewußtsein doch wieder zusammenzusetzen, davon ist gar keine Rede. Was bedeutet denn WundTs Aufstellung der „Apperzeption"? Doch nichts anderes, als daß alle Mühen der Assoziationspsychologie vergeblich gewesen sind, und daß, wer mit seelischen Elementen arbeitet, schließlich immer noch eine wirkliche Seele einführen muß - nur daB man dann nicht, Seele", sondern eben „Apperzeption" zu ihr sagt.

Schon vor mehr als zwanzig Jahren hat v. Kries an den anatomisch-physiologischen Grundlagen der Assoziationspsychologie eine bisher niemals widerlegte und meines Erachtens gar nicht widerlegbare Kritik geübt. Inzwischen haben die Ergebnisse der Hirnpathologie, die seinen Anschauungen damals noch zu widersprechen schienen, sie zu stützen begonnen ${ }^{1}$ ) - auch sie haben jede Hoffnung vernichtet, daß uns die Natur in der Symptomatologie der organischen Psychosen etwa die Bausteine in die Hand spielen würde, aus denen sich das Seelenleben des Gesunden konstruieren ließe. Schließlich aber, und das ist das Wichtigste, haben die psychologischen Arbeiten von KÜLPE, MESSER, BÜHLER, ACH und anderen sowie kritische Überlegungen von R. HoEnIGsWAID die grundsätzliche Unmöglichkeit aller ,Atomisierungs"versuche dargetan und gezeigt, daß sich das menschliche Denken durch das Spiel von Gleichzeitigkeits- und Ähnlichkeitsassoziationen, von Konstellationen und Zielvorstellungen niemals aufklären läßt.

Zugleich hat uns diese moderne Denkpsychologie eine Erkenntnis vermittelt, die sich wieder mit den herkömmlichen Ansichten der Assoziationspsychologie ebensowenig verträgt wie mit den anatomisch-physiologischen Grundanschauungen des Mediziners, geschweige denn mit den psychiatrischen Folgen, die WERNICKE aus seiner Aphasielehre abzuleiten versucht hatte. Das ist die Einsicht, daß es ein Denken ohne Sprache gibt, daß wir neben dem anschaulichen Teil unseres Denkens, der sich in Vorstellungen und gedachten Worten abspielt, noch einen unanschaulichen, weder von sonstigen Erinnerungsbildern noch vom Wort getragenen Teil anerkennen müssen. Namentlich bei gefühlsbetonten Gedanken wird die Klarheit, die ihnen das auch nur gedachte Wort verleiht, überaus häufig vermieden. Wieder ist so eine Schranke gefallen, die verschiedene seelische "Elemente" trennen sollte; denn Gedanken, die ohne jede sinnliche Komponente und sogar ohne sprachliche Fassung ins Bewuitsein treten, lassen sich von ,Gefühlen" schwer unterscheien.

Dafür sind durch diese Einsicht Bewußtseinsvorgänge ins Licht der wissenschaftlichen Betrachtung gerückt, die bis dahin nur der unwissenschaftlichen Laienpsychologie bekannt und die von der Assoziationspsychologie aus guten Gründen stets ignoriert worden waren. Es ist kein Zufall, daß sich die Denkpsychologie in den Berichten über die ,"Ver* suche", die auch sie anstellt, die aber mit den Experimenten des physiologischen Laboratoriums gar nichts gemein haben,

1) So schreibt MONAKOW: DaB die

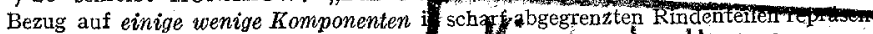

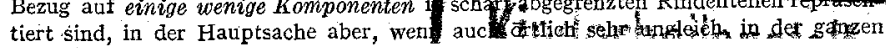
Rinde.: 
der. gewöhnlichen Umgangssprache bedient. Da ist nicht mehr von Assoziationen und Konstellationen die Rede, von psychischer Energie und von Vigilität der Aufmerksamkeit, von Ziel- und Obervorstellungen, sondern in der ihnen geläufigen Sprache des täglichen Verkehrs drücken die Versuchspersonen möglichst naiv das aus, was sie unmittelbar seelisch erleben. Damit ist nicht bloß die Bedeutung gewürdigt, die der Sprache eines Menschen für die Beurteilung seiner Persönlichkeit zukommt, sondern zugleich endlich auch das anerkannt, daß wir weder ohne Selbstbeobachtung noch ohne die Psychologie des täglichen Lebens auskommen, daß nicht die ,Psychologie der Elemente", sondern nur eine Psychologie, die das komplexe seelische Erlebnis berücksichtigt, all die Fragen zu beantworten vermag, über die wir, wenn von der Seele die Rede ist, beim gesunden wie beim kranken Menschen schließlich wirklich Auskunft verlangen. Diese Psychologie aber ist - wenn auch wissenschaftlich geformt und wissenschaftlich ausgebaut - die Psychologie des täglichen Lebens, die Psychologie der Dichter und Geschichtsschreiber, der Diplomaten, Seelsorger und Arzte; ja sie ist auch immer schon die Psychologie der Psychiater gewesen - nur hat man sich hier ihrer beinahe immer geschämt. Fast alles, was wir an tiefen und feinen Gedanken über den gesunden Menschen in der Literatur finden, steht in Selbstbekenntnissen, Autobiographien und Briefen, in Romanen, Gedichten und Dramen, in philosophischen Essays oder in historischen, kultur-oder kunstgeschichtlichen Arbeiten, und sehr brauchbare Ansätze zur Erklärung gewisser pathologischer Vorkommnisse ${ }^{1}$ ) liegen bei Dostojewski, IBSEN und StrindberG, um nur einige wenige herauszugreifen.

Für einen jungen Psychiater aber hätte es noch vor zehn Jahren eines nicht gewöhnlichen Mutes bedurft, um Psychologie in diesem Sinne nicht nur zu treiben, sondern auch darüber zu schreiben. Nur in unseren Krankengeschichten und in klinischen Arbeiten haben wir sie niemals ganz entbehren können. Wäre auch hier nur von Assoziationen die Rede gewesen, so hätten wir uns noch schlechter untereinander verständigt und noch weniger von dem erfahren, was unsere Kranken innerlich wirklich erleben. Man kann ruhig zugeben: die scharfsinnigen Bemerkungen über psychopathologische Zustände sowohl, die uns bei WERNICKE immer wieder erfreuen, wie die großen, klar und tief verstandenen psychischen Krankheitsbilder, die wir KRAEPELIN verdanken, haben mit der wissenschaftlichen Psychologie, auf die sich die Lehrgebäude beider Forscher scheinbar stützen, schlechthin gar nichts zu tan. Wundts Psychologie ist ja bei KRAEPELIN überhaupt nichts als ein lose untergeschobener, mit den oberen Stockwerken organisch durchaus nicht verbundener Unterbau, der jeden Augenblick durch einen anderen ersetzt werden könnte.

Bei WERNICKe liegen die Dinge wohl anders; aber hier können wir auch deutlich verfolgen, wie sehr die grundsätzliche Einstellung geschadet und gehemmt hat. Warum ist der glückliche Wurf der überwertigen Ideen so lange beinahe ohne jede klinische Wirkung geblieben und warum hat sich ein so fruchtbarer Gedanke erst in Gestalt der FREUD schen "Komplexe" durchsetzen können? Weil man zu WERNICKEs Zeiten das Seelische atomisierte, weil man im Grunde gar nicht auf die Psyche und den lebendigen Menschen, sondern auf das Gehirn eingestellt war und nicht nach Uberzeugungen, Stimmungen und Wünschen, sondern nach ihren anatomischen Grundlagen forschte. So erhielt damals jedes seelische Symptom bei der wissenschaftlichen Behandlung beinahe automatisch ein neurologisches Gesicht. Gewisse Arbeiten über die Entstehung der Sinnestäuschungen und des Wahns, über die autochthonen und die $Z$ wangsideen, über epileptische Dämmerzustände usf., die aus dieser Zeit stammen, handeln, bei Lichte besehen, gar nicht von den unmittelbaren psychologischen Erfahrungen der Klinik; erst wenn diese Erfah-

1) Das soll natü-lich nicht heißen, daß die Psychosen, die gelegentlich die Dichter anf die Bühne bringen - wie die Gretchens im Faust oder die Ophelia's Dichter anf die Bühne bringen - wie die Gretchens im Faust oder die Ophelia's
z. B. - irgendwelches psychiatrisches Interesse besäßen. Diese Szenen lehren ja doch höchstens, was sich die Dichter unter einer Psychose vorgestellt haben, und daraus karn jemand, der selbst täglich Psychosen sieht, doch wirklich nichts lernen. rungen in die neurologische Sprache des Autors übersetzt und seinen anatomisch-physiologischen Grundanschauungen angepaßt waren, setzte die eigentliche gedankliche Verarbeitung ein.

Dicse Zeit liegt weit hinter uns; aber auch die ist abgeschlossen, in der psychologische Beobachtungen erst dann als wissenschaftlich galten, wenn sie in Kurven oder Diagrammen niedergelegt waren. Zugleich hat sich der Schwerpunkt unseres Interesses verschoben. Wir erwarten für die Psychiatrie von der ,"Psychologie der Elemente " nichts mehr und wir bekennen uns mit Nachdruck zu einer psychologischen Arbeitsrichtung, die die normalen wie die pathologischen Bewußtseinșerscheinungen nicht mehr zu zerpflücken, sondern als Ganzes zu verstehen versucht.

Auch von seinen Gegnern soll man lernen. Der ungeheure Erfolg der FREUD schen Schule - wir wollen uns nicht darüber täuschen, daß dieser Erfolg ein ungeheurer gewesen ist - ist nur dadurch möglich geworden, daß die offizielle Wissenschaft gar so wirklichkeitsfremd war, daß sie von den tatsächlichen seelischen Erlebnissen scheinbar so sehr wenig wußte, und daß sie dem, der wirklich von der ,Seele" etwas $\mathrm{zu}$ erfahren wünschte, Steine reichte anstatt Brot. Die Freudsche Schule enthält so viel Abstoßendes; die Kritiklosigkeit, mit der phantastische Eingebungen des einzelnen als objektive Wahrheiten ausgegeben, die spitzfindige Dialektik, mit der die unmöglichsten Gedankensprünge möglich gemacht, die Skrupellosigkeit, mit der ohne jeden Beweis Hypothesen auf Hypothesen getürmt werden, und nicht zuletzt die ungeheure Uberschätzung geschlechtlicher Motive hätten sie längst um alles Ansehen gebracht, wenn sie nicht die einzige psychologische Richtung gewesen wäre, die das Seelische als Ganzes zu erfassen versuchte.

Jetzt liegen die ersten Versuche vor, unabhängig von FREUD, befreit aber auch von allem Ballast der ,Hirnmythologie" und der konventionellen Atomisierung der Seele, komplexe psychische Vorgänge bei Gesunden und Kranken zum Gegenstand einer gründlichen, kritischen und doch lebensnahen Untersuchung zu machen. Von allem anderen abgesehen haben unsere neugewonnenen Erkenntnisse uns ja auch gewisse Tiefen der Seele wieder erschlossen, zu denen der Zugang bisher durch das Schlagwort des ,unbewußten seelischen Geschehens" verwehrt worden war. Auch an diese Unterseele, die hinter den Kulissen die Hebel stellt, die die menschlichen Uberzeugungen und Handlungen nach geheimnisvollen, nur der Psychoanalyse zugänglichen Gesetzen und aus ebenso mystischen Motiven bestimmt, auch an sie glauben wir nicht. Was von Freuds ,Tiefenpsychologie" übriggeblieben ist, geht in dem auf, was wir als "Gefühlspsychologie" bezeichnen; das "Unbewußte" aber - soweit es sich um ein psychisch Unbewußtes und nicht um eine metaphysische Annahme handelt - ist der Inbegriff all der peinlichen, nicht eingestandenen und doch mächtigen inneren Strebungen, die wir nicht kennen wollen ${ }^{x}$ ) und die wir zum mindesten vor anderen so viel wie nur möglich verbergen, die man aber - leider - bei sich selbst doch nur allzu gut kennt. Im Assoziationsexperiment freilich und im physiologischen Laboratorium wird man dem Untersucher davon gewiß nichts verraten.

Und jetzt wird man fragen, was denn nun geschehen solle und was schon geschieht. Wir sind dazu übergegangen, beim Gesunden und Kranken komplexe seelische Zustände und Vorgänge, gewisse Einstellungen und Realtionen, wie Eifersucht, Mißtrauen, Eigensinn, Eigennutz und Ehrgeiz, Selbstbewußtsein und Schüchternheit - um wahllos einiges herauszugreifen - zu studieren, und wir werden zugleich scharf umrissene Typen, und $z$ war wieder auf dem normalen wie auf dem krankhaften Gebiet, aufzustellen versuchen. Die ersten Ansätze dazu liegen bereits vor; daß der wertvollste auf normalem Gebiet - in EDUARD SPRANGERS , Lebenstormen"'2) -

1) GORKI schildert es einmal als die einzige Hölenstrafe, dab man alles sehen wercle, was man bei Lebzeiten vcr sich selber verborgen gehalten hätte.

2) Sehr charakteristisch ist, daß SPRANGER am besten zweifellos die Analyse des "theoretischen Menschen" gelungen ist. Der theoretische Mensch, das ist eben des "theoretischen Menschen" gelungen ist. Der theoretische Mensch, das ist eben
der Gelehrte, den der Gelehrte natïrlich am besten kennt und am lebendigsten zu schildern vermag. 
von einem Philosophen stammt, darf uns nach der bisherigen Entwicklung der Dinge weder wundern noch gar verstimmen. Aber auch die Psychiatrie hat, nachdem Hocre, JAsPERS, BIRNBAUM u. a. die Wege gewiesen hatten, verheißungsvolle Anläufe gemacht, die großen durch alle moderne Kritik unangetasteten Bilder der KRAEPELIN schen Systematik psychologisch näher auszuführen und dabei die Umrisse der ursprünglichen, durch die Krankheit nur veränderten Persönlichkeit noch stärker hervortreten zu lassen. Auch hier werden wir uns der Sprache des Alltags bedienen, wir werden die Mittel des Schriftstellers, des Feuilletonisten und des Dichters benützen, genau so wie wir gewissen hervorragenden Vorbildern unter den Historikern, den Literatur- und Kunsthistorikern nacheifern werden. Wir werden auch hier nicht blutleere, abstrakte Schemata konstruieren, sondern lebendige Menschen hinzustellen versuchen, obwohl oder vielleicht gerade weil wir wissen, daß kein Mensch, auch kein kranker Mensch dem anderen vollkommen gleicht. Denn wir haben eingesehen, daß die bloße Häufung von Eigenschaften, die verschiedenen Persönlichkeiten entlehnt sind, selbst wenn sich diese noch so sehr ähneln, das gesamte entstehende Bild notwendig unwirklich und farblos gestaltet.

So stehen wir in der Psychologie sowohl wie in der klinischen Symptomatologie im Beginn einer neuen Epoche, die überaus fruchtbar werden könnte. Aber schon im Entstehen drohen dieser neuen Richtung schwere Gefahren, Gefahren, die diesmal nicht von irgendwelchen Gegnern, sondern im Gegenteil von besonders überzeugten Anhängern ausgehen. Es sind in letzter Zeit mehrfach recht kriegerisch klingende Stimmen laut geworden, die die Psychologie wie die Psychiatrie philosophisch zu fundieren versuchen. Dagegen wäre an sich nichts $\mathrm{zu}$ sagen, wèn nicht auch dabei wieder eine wirklichkeitsfremde Grundauffassung zutage träte, die lebhaften und sehr berechtigten Widerspruch hervorgerufen hat. Man versucht die Psychiatrie aus der Gesamtmedizin herauszulösen und als Zweig der Geisteswissenschaften neu zu begründen. Man fordert dementsprechend die Schaffung einer "reinen Psychiatrie", die jede Einstellung auf das Gehirn und den Körper überhaupt und somit jede Befassung mit anatomischen, neurologischen und serologischen Fragen abschwören soll. Schon vor bald zehn Jahren hat W. SpEchт Ahnliches gefordert, jetzt aber werden diese Forderungen in sehr apodiktischer Weise wiederholt. Unsere hervorragendsten Kliniker werden beschuldigt, ihre eigene Lehre dadurch verwässert und verdorben $\mathrm{zu}$ haben, da $\beta$ sie auch "heterologische" Arbeitsweisen neben der rein psychiatrischen zugelassen oder gar ausgebaut hätten.

Man wird nicht leugnen dürfen, daß diese Forderungen gefährlich sind. Wir würden heute ja vermutlich von der Paralyse noch weniger wissen als von der Dementia praecox, wenn uns jede ,Einstellung" auf das Gehirn und den sonstigen Körper schon vor hundert Jahren mit ähnlicher Entschiedenheit verboten worden wäre wie jetzt. Und mit der Entgegnung, die Paralyse als "Gehirnkrankheit" ginge die Psychiatrie eben gar nichts mehr an, werden wir uns auch heute noch nicht beruhigen wollen. Nun sind gewiß manche Arbeiten dieser Art sehr harmlos und mehr als logische Stilübungen $z u$ werten: sie werfen die organischen Krankheiten zum psychiatrischen Tempel hinaus und lassen sie dann - als symptomatische Psychosen - zur Hintertür wieder hinein. Immerhin, wenn ein Forscher vom Range A. PICks am Ende seiner alkademischen Wirksamkeit und als Abschluß ungewöhnlich großer klinischer Leistungen es für notwendig hält, in einer methodologischen Studie die neurologische Forschungsrichtung in der Psychiatrie ${ }^{1}$ ) zu begründen und zu rechtfertigen, dann muß diese Richtung doch wohl schon lange nicht mehr die Würdigung finden, die sie nicht bloß ihrer geschichtlichen Leistungen - erst sie hat uns von HeINROTHS moralisierender Mystik befreit -, sondern auch ihrer zukünftigen Aufgaben wegen verdient.

Für noch bedenklicher aber halte ich es, daß die erwähnten Kritiken des Alten und die Forderungen, die auf ihnen basieren, gerade die psychologische Arbeitsweise in Miskredit 1) Berlin, Karger, xgar. bringen könnten. Erheben lassen sich solche Forderungen ja leicht - genau so leicht, wie in der Politik die Opposition Programme aufstellen kann, vor deren Verwirklichung die Verteilung der Machtverhältnisse diese selbe Opposition auf unabsehbare Zeit schützt. Man stelle sich vor, was die überzeugtesten Vertreter der ,reinen" Psychiatrie wohl sagen würden, wenn eine nur psychologisch orientierte Klinik für psychische Krankheiten wirklich einmal geschaffen werden sollte. Ich vermute, es würde ihnen gehen wie jenem Dichter, dessen Geliebten ein boshafter Gott wirklich Korallenlippen und Marmorhände verlieh.

Der Humor von der Sache ist, daß alle diese philosophierenden Auswüchse in letzter Linie auf einem Mißverständnis beruhen. Sie berufen sich alle auf HusserLs ,Phänomenologie . Daß gerade HUSSERL ${ }^{1}$ ) der Naturwissenschaft dringend empfiehlt, ,die Grenzen der dogmatischen Forschung gegenüber von kritizistischen Fragestellungen abzuschließen " und sich durch ,erkenntnistheoretische Vorurteile, über deren Recht oder Unrecht die Philosophie zu entscheiden habe", im Gang ihrer Forschungen nicht hemmen zu lassen, wird dabei ebenso übersehen wie Husserls mehrfach abgegebene Erklärung, „daß die reine Phänomenologie nicht Psychologie ist"; ,daß nicht zufällige Gebietsabgrenzungen und Terminologien, sondern prinzipielle Gründe es ausschließen, $\mathrm{da} \beta$ sie der Psychologie zugerechnet werde; daß endlich die Psychologie eine Erfahrungswissenschaft, eine Wissenschaft von Tatsachen, von Realitäten, daß dagegen die Phänomenologie eine Wissenschaft ist, die ausschließlich, „Wesenserkenntnisse "feststellen wolle, ,und durchaus keine Tatsachen".

Wenn man also heute in der Psychologie und Psychiatrie von phänomenologischer Forschung spricht, so kann das zwei ganz verschiedene Dinge bedeuten: entweder eine philosophische Fundierung der Psychologie, die, an sich wohl notwendig and erwünscht, von der psychologischen und psychiatrischen Tatsachenforschung gerade nach HusserL nicht abgewartet zu werden braucht; oder aber etwas, was mit Husserls "Phänomonologie" wirklich nur den Namen gemein hat: das für uns heute wieder selbstverständliche Bestreben nämlich, zunächst zu erfahren, was ist, was sich im Bewußtsein von Gesunden und Kranken denn eigentlich abspielt.

Das ist zunächst reine Tatsachenforschung. DaB die so zutage geförderten Tatsachen dann sofort begrifflich und systematisch geordnet werden müssen, das versteht sich grundsätzlich ja wohl für jede Wissenschaft von selbst; daß es in der Psychiatrie darin recht oft gehapert, und daß uns in dieser Hinsicht namentlich JASPERS durch die philosophische Schulung, die er in die Behandlung psychiatrischer Fragen hineintrug, ganz wesentlich gefördert hat, das werde gerade ich als letzter bestreiten. Aber zur reinen Geisteswissenschaft wird die Psychiatrie dadurch nicht - wer von den Jüngeren das mir nicht glaubt, den darf ich vielleicht auf sehr lesenswerte kritische Uberlegungen wieder eines Philosophen, R. HoEnIGsWAlds ${ }^{2}$, verweisen. Nach ihm ist die Psychiatrie, ,dem methodischen Sinn ihrer Fragestellung, der Struktur ihrer Begriffsbildung und Begriffsgliederung nach Naturwissenschaft". Aber selbst die Psychologie, die derselbe Forscher an sich von der Naturwissenschaft trennt, kann sich ,,grundsätzlich und in der ganzen Ausdehnung ihrer Problemkreise" keineswegs „,von jeder Rücksicht auf physiologische Aufgaben frei machen".

Wir haben doch eigentlich keinen Grund, päpstlicher zu sein als der Papst. Die Neigung dazu ist aber recht groß auch innerhalb der gerichtlich-medizinischen Literatur ist mir immer aufgefallen, daß die verzwicktesten juristischen Tifteleien nicht von Juristen, sondern von Medizinern herrührten. In der Philosophie ist es ganz ähnlich. Auch wer, wie ich, die innigen Beziehungen der Psychologie und der Psychiatrie zu den Geisteswissenschaften dauernd im. Auge behält und deshalb jeden ernsten Versuch, die allgemeine Stellung der Psychologie und Psychiatrie im System deI

1) Ideen zu einer reinen Phaenomenologie und phaenomenologischen Philosophie,
Halle a. d. Saale, Niemeyer rgr3.

2) Die Grundlagen der Denkpsychologie, Reizhardt, München, rg2I. 
Wissenschaften neu zu umgrenzen ${ }^{1}$ ), persönlich aufs wärmste begrüßt, wird es anderen Fachgenossen ${ }^{2}$ ), nicht verargen dürfen, wenn sie sich angesichts mancher dilettantischer Versuche, die gerade in unserer Literatur vorliegen, gegen jeden Einbruch der Philosophie in die Psychiatrie grundsätzlich und mit Entschiedenheit wehren. In keinem Falle aber werden wir kritizistischen Ubungen dieser Art erlauben wollen, uns hinsichtlich unserer praktischen Arbeit Vorschriften zu machen. Wir werden ruhig das Neue betreiben, ohne das Alte zu verketzern und abzuschwören. Wir werden das Wesen und die Erscheinungen der Geisteskrankheiten von allen Seiten, von der anatomischen, neurologischen und serologischen sowohl wie von der psychologischen zu studieren versuchen, und wir werden uns überdies bei jedem Spezialgebiet, das wir selbst wählen, bewußt bleiben, daß seine theoretischen Voraussetzungen, so wie sie uns richtig erscheinen, sicher nicht die sein werden, die eine künftige Generation anerkennen und übernehmen wird.

\section{ORIGINALIEN.}

\section{DIE NATÜRLICHEN ABWEHRMITTEL DES KÖRPERS GEGEN DIE SYPHILITISCHE INFEKTION UND IHRE BEEINFLUSSUNG BESONDERS DURCH QUECKSILBER.}

Von

\author{
Dr. S. BERGEL, Berlin.
}

In früheren Arbeiten $^{2}$ ) habe ich gezeigt, daß die syphilitischen Erscheinungen, die anatomisch sich im wesentlichen als entzündliche lymphocytäre Infiltrationen präsentieren, eine nach chemotaktischen Gesetzen sich vollziehende Folge der Spirochäteninfektion, und somit zwar ein Krankheitsprodukt sind, aber gleichzeitig eine natürliche, wenn auch oft unzureichende Abwehrreaktion des Organismus darstellen, die chemisch auf einem Abbau der lipoiden Spirochäten durch die spezifisch eingestellten lipolytischen Lymphocyten beruht, und die serologisch ihren Ausdruck in dem Vorhandensein einer positiven Wassermannschen Reaktion findet. Infolge dieser neugewonnenen Erkenntnisse war überhaupt erst die Möglichkeit gegeben und eine Grundlage geschaffen, auf der man experimentell und klinisch die Frage beantworten konnte, ob ein Heilmittel die natürlichen Abwehrvorgänge des Organismus unterstützt oder verhindert; man wußte eben vorher nicht, selbst wenn man schon annehmen wollte, daß jede „Entzündung" auf alle Krankheitsprozesse irgendwie heilend wirke, was in dieser Allgemeinheit nicht richtig ist, welcher Bestandteil des Entzündungskomplexes gerade bei der Lues das wesentlich heilsame Agens darstelle, aus welchem biologischen Grunde er das sei, und ob, bezw, in welchen Beziehungen diese Vorgänge zur Wassermannschen Reaktion stehen.

LESSER $^{4}$ ) hat nun gesagt, daß das Quecksilber ein Mittel seí, das dem natürlichen Heilungsprozeß entgegenarbeite; das lediglich die Krankheitssymptome beseitige, ohne die Spirochäten zu beeinflussen, und glaubt dafür klinische Belege beigebracht zu haberi. So gewichtig manche auch sein mögen, z. B. die sehr seltenen Reinfektionen nach Quecksilberbehandlung im Gegensatz zu denen nach Salvarsan, so sind doch andere nicht als ${ }^{2}$ wingend und eindeutig anzuerkennen. Vom rein klinischen Standpunkte aus $\mathrm{zu}$ dieser Frage entscheidend Stellung zu nehmen;; steht mir nicht zu, und dürfte auch kaum zu einem Resultate führen, wie die divergierenden Ansichten unter den Klinikern früher und jetzt beweisen.

Aber auf Grund unserer neuen Anschauungen, die uns die biologische Bedeutung der klinischen, Erscheinungen, sowie der anatomischen und serologischen Befunde bei der Syphilis so ungezwuingen und überraschend einfach dem Verständnis nähergebracht haben, will ich versuchen, zur Klärung dieser praktisch sehr wichtigen und wissenschaftlich interessanten therapeutischen: Frage einen Beitrag zu liefern.

Ein gesicherter Beweis, wie das Quecksilber bei der Syphilis wirkt, liegt bisher nicht vor; feststehend ist aber die

\footnotetext{
1) Vergl. A. HOPPE und namentlich R, HOENIGSWALD.

2) Vergl. BLEULER und STRANSKY.

3) Münch. med. Wochenschr. I909 Nr. 2; I9ro Nr. 32; Igr2 Nr. 20; I92I Nr. 36; und Die Lymphocytose Berlin r 921 bei Julius Springer.

7) Dtsch. med. Wochenschr. Ig2I Nr. 2-3; Berl. klin. Wochenschr. I92I Nr. 46.
}

Tatsache, daß es einen Einfluß hat, wahrscheinlich auch auf den Krankheitsverlauf, zum mindesten aber auf die klinisch sichtbaren syphilitischen Krankheitserscheinungen. Wo setzt nun diese Wirkung ein, und wie ist sie zu erklären?

Nach den experimentellen Untersuchungen und klinischen Erfahrungen erscheint es sehr zweifelhaft, wenn nicht ausgeschlossen, daß das Quecksilber in der gebräuchlichen Dosierung die Syphiliserreger unmittelbar und vollständig abtötet, da die Spirochäten tötende Dosis bereits eine für den menschlichen Organismus giftige ist. Es ist also anzunehmen, daß sein Einflu $\beta$, falls er überhaupt, wenn auch unzureichend, sich auf die Spirochäten erstrecken sollte, über die natürlichen Mittel des Körpers geht, oder daß er ein ausschließlich symptomatischer, lediglich die sichtbaren Krankheitsprodukte betreffender ist. In beiden Fällen erhebt sich die Unterfrage, welcher Art dieser Einfluß ist.

In dieser Hinsicht erscheint es von Wichtigkeit, daß eine spezielle Einwirkung des Quecksilbers auf lymphoides Gewebe anscheinend sichergestellt ist. Nachdem wir, wie gesagt, jetzt wissen, daß die Lymphocyten und ihre Bildungsorgane, die Lymphdrüsen, die Antistoffbildner gegenüber den Spirochäten sind, daß diese Schutz- und Heilwirkung auf einer spezifischen Lipasenwirkung gegenüber dem Lueslipoid beruht, deren biologischer Ausdruck die Wassermannsche Reaktion ist, ist vielleicht gerade in den Beziehungen des Quecksilbers zu dem für die syphilitischen Krankheitsprodukte so charakteristischen lymphoiden Gewebe der Schlüssel zur Lösung der Frage nach seiner Wirkung überhaupt zu finden.

Am besten läßt sich der Einfluß der Quecksilberbehandlung anatomisch und klinisch beim Primäraffekt verfolgen, und hier werden wir auch am ehesten imstande sein, eine biologische Deutung des Heilprozesses zu geben.

LESSER selbst kennzeichnet den Unterschied zwischen der Salvarsan- und der Queckilberwirkung beim Primäraffekt nach den Untersuchungen von STEJSKAI dadurch, daß nach Salvarsaninjektionen die chemotherapeutisch getroffenen Spirochäten plötzlich verschwinden, und daß das Infiltrat sich allmählich zurückbildet, während nach Quecksilbereinwirkung allmähliches Schwinden der Spirochäten aus dem Primäraffekt entsprechend der Rückbildung des Infiltrates erfolgt.

Wenn wir diesen Befund, und ähnlich dürfte er auch bei anderen syphilitischen Manifestationen sein, von unserem Gesichtspunkte aus biologisch analysieren, so ergibt sich ungezwungen folgende Erklärung. Die Spirochäten werden durch das Salvarsan direkt primär abgetötet, ihre z.T. lipoiden Zerfallsprodukte werden von den dadurch vielleicht noch vermehrten Reaktionszellen, den lipolytischen Lymphocyten, aufgenommen bzw. weiter abgebaut. Wenn sie diese Funktion erfüllt haben, und wenn Spirochäten nicht mehr vorhanden sind, verschwinden sie naturgemäß, da die Ursache ihres Auftretens beseitigt ist. Das Quecksilber dagegen tötet die Spirochäten nicht unmittelbar ab; daher kommt es auch nicht zu einem plötzlichen Verschwinden derselben, sondern das Quecksilber wirkt hier auf die lymphoide Umwallungszone, die der Körper als Abwehrreaktion gegen die Spirochäten aufgerichtet hat, und zwar, nach den anatomischen, serologischen und klinischen Befunden zu urteilen, derart, daß immer ein Teil der Lymphocyten zerfällt, was zur Folge hat, daß einerseits das Infiltrat allmählich kleiner wird, und daß 